Revue d'histoire de l'Amérique française

REVUE D.HISTOIRE DE L'AMÉRIQUE FRANÇAISE

\title{
Les Canadiens ont-ils payé la dîme entre 1760 et 1775 ?
}

\section{Georges-Étienne Proulx}

Volume 11, numéro 4, mars 1958

URI : https://id.erudit.org/iderudit/301862ar

DOI : https://doi.org/10.7202/301862ar

Aller au sommaire du numéro

Éditeur(s)

Institut d'histoire de l'Amérique française

ISSN

0035-2357 (imprimé)

1492-1383 (numérique)

Découvrir la revue

Citer cet article

Proulx, G.-É. (1958). Les Canadiens ont-ils payé la dîme entre 1760 et 1775 ?

Revue d'histoire de l'Amérique française, 11(4), 533-562.

https://doi.org/10.7202/301862ar d'utilisation que vous pouvez consulter en ligne.

https://apropos.erudit.org/fr/usagers/politique-dutilisation/ 


\section{LES CANADIENS ONT-ILS PAYÉ LA DÎME ENTRE 1760 ET 1775? *}

Le changement d'allégeance du Canada français en 1760 a créé, du point de vue religieux, des situations fort complexes. Le passage d'une domination catholique à un régime protestant, intolérant envers l'Église catholique, ne pouvait que susciter de graves problèmes et causer chez notre peuple de sérieuses inquiétudes concernant l'avenir de sa religion et des institutions qui en assuraient l'existence.

On sait généralement ce qu'il advint de la dîme, au point de vue légal, en 1760 ; mais on ignore presque totalement quelle fut l'attitude des Canadiens à l'égard de cette obligation religieuse, durant cette période d'incertitude qui se prolonge jusqu'à l'année 1775.

C'est donc à une question très précise que nous essaierons de répondre: "Les Canadiens, entre 1760 et 1775, ont-ils continué de payer la dîme à leurs curés ? » Il s'agit pour nous de vérifier un fait, et non pas de définir une situation légale; il convient cependant, pour mieux situer le sujet, de le placer dans son contexte légal, et c'est pourquoi nous verrons d'abord quel était le

* Thèse présentée à l'Institut d'Histoire de l'Université Laval, pour l'obtention de la licence d'histoire.

Sigles et abréviations utilisés dans ce travail:

AAQ Archives de l'Archevêché de Québec.

AEP Archives de l'Evêché de Ste-Anne de la Pocatière.

ANDQ Archives de la Paroisse Notre-Dame de Québec.

Doc. Const. Documents pour servir à l'histoire constitutionnelle du Canada, 1759-1791.

EO Edits et Ordonnances royaux.

MEQ Mandements, lettres pastorales et circulaires des évêques de Québec.

MSRC Mémoires de la Société Royale du Canada.

RAPQ Rapport de l'Archiviste de la Province de Québec. 
statut légal de la dîme à la fin du régime français, et quel fut le changement apporté à ce statut par la capitulation de 1760 .

C'est par l'acte d'érection du Séminaire de Québec, ${ }^{1}$ en 1663 , que fut aussi établie la dîme au Canada; l'habitant devait payer à son curé, ou au desservant de sa paroisse ou mission, la treizième partie de ses récoltes de grains, c'est-à-dire, blé, sarrazin, maïs, orge, seigle, avoine, pois. ${ }^{2}$

Cette loi cependant ne fut mise en force qu'en 1667, par une ordonnance, édictée à la demande de Mgr de Laval par Tracy, Courcelle et Talon. ${ }^{3}$ Sur les représentations des habitants, et vu la pauvreté du plus grand nombre d'entre eux, on avait réduit la quotité au vingt-sixième minot; cette réduction, qui devait être temporaire, fut sanctionnée, en 1679, par une nouvelle ordonnance qui la fixait ainsi définitivement à la vingt-sixième portion. ${ }^{4}$

La dîme était due par tout propriétaire ou fermier, à proportion du profit retiré par chacun d'eux; ${ }^{5}$ elle n'était pas due, sur les terres nouvellement défrichées, pendant les cinq premières années ; ${ }^{6}$ de plus elle devait être portée au lieu de résidence du curé, après avoir été battue et vannée. ${ }^{7}$ Ajoutons à cela que les curés dont la dîme n'était pas suffisante pour les faire vivre con-

1 Etablissement du Séminaire de Québec par M. l'Evêque de Pétrée, 26 mars 1663, dans Edits et Ordonnances, 1: 34.

2 Approbation du Roi pour l'établissement du Séminaire de Québec, avril 1663, ibid., 36. Un canoniste mentionne aussi les vesces parmi les grains décimables. Cf Desautels, Manuel des curés, 89.

3 L'original de ce règlement resta au secrétariat de l'intendant Talon et fut perdu. Il en est cependant fait mention dans une ordonnance de 1679 et une autre de 1705. Cf Mignault, Droit paroissial, 150-155.

4 Edit du Roi concernant les Dîmes et Cures fixes, 23 octobre 1679, dans EO, 1: 231s.

5 Règlement du Conseil Supérieur pour le Payement des Dîmes par le propriétaire et par le fermier, du 20 mars 1668, dans EO, II : 45.

6 Ordonnance du 23 août 1667, citée par Baudry, Code des Curés, 77ss. Cette copie de l'ordonnance perdue de 1667, a été trouvée par Baudry; Mignault dit qu'on n'a aucune preuve que cet article concernant les terres nouvelles ait jamais été appliqué. Mignault, op. cit., 173.

7 Ordonnance du 23 août 1667, Baudry, op. cit., 77ss. et aussi Ordonnance du 27 mars 1713, dans EO, III: 158. 
venablement, pouvaient se pourvoir auprès du Conseil supérieur et se faire octroyer une part des 8,000 livres fournies annuellement par le roi à cet effet à partir de $1682 .{ }^{8}$

Comme le fait remarquer Mignault, toute cette législation porte sur la quotité et le mode de perception des dîmes, et aucunement sur l'obligation même de payer la dîme, parce que cette obligation était de droit commun. ${ }^{9}$

Mais ce qu'il faut surtout remarquer, c'est l'étroite collaboration qui existe entre les autorités civiles et religieuses: tous les édits que nous avons mentionnés émanent du pouvoir civil, le Conseil supérieur, dont l'évêque sans doute est l'un des principaux membres, mais qui est quand même l'organisme qui dirige l'administration civile de la colonie; de plus on retrouve souvent, dans les jugements du Conseil, des arrêts ordonnant à tel paroissien de payer sa dîme, sous peine d'une amende, qui est d'ordinaire de dix livres. ${ }^{10}$

La dîme était donc non seulement une loi ecclésiastique, mais aussi une loi que l'autorité civile avait faite sienne, et dont on pouvait réclamer l'application devant les cours civiles de justice.

Ce système n'était guère possible que dans un pays officiellement catholique, comme la France et la Nouvelle-France; il semble même que les protestants devaient se soumettre en France à la même obligation que les catholiques: la législation française ne distinguait pas entre catholiques et protestants, et tous ceux qui cultivaient la terre, quelle que fût leur croyance, devaient payer la dîme au curé catholique.

On conçoit dès lors qu'une telle législation pouvait difficilement être acceptée par un vainqueur qui refusait chez lui de reconnaître l'Église catholique romaine, et c'est pourquoi la con-

${ }^{8}$ Gosselin, Mgr de Laval, I: 414s. Sur cette somme de 8,000 livres, 2,000 servaient pour les prêtres vieux ou malades, et 1,200 pour la construction d'églises; le reste, soit 4,800 livres, était partagé entre les curés dont la dîme était insuffisante. Dans une lettre écrite par le curé $\mathrm{Ph}$. Boucher au gouverneur, il est fait mention d'un montant de 1,600 livres seulement affecté au supplément des dîmes: Cf J.-Edmond Roy, Histoire de la Seigneurie de Lauzon, vol. 2, appendice V: XVII.

9 Mignault, op. cit., 157, note 2.

$10 \mathrm{EO}, \mathrm{II}: 309 \mathrm{~s}, 434,484 \mathrm{~s}, 513,516$; III: 158s, 174, 175. 
quête devait modifier profondément le statut légal de la dîme au Canada, et même le faire disparaître.

Dans le traité de capitulation de Montréal, le gouverneur Vaudreuil avait proposé au vainqueur la clause suivante:

Ces peuples [du Canada] seront Obligés par le Gouvernement Anglais à payer aux prestres qui en prendront soin, Les Dixmes et tous les droits qu'ils avaient Coutume de payer sous le Gouvernement de Sa Majesté très Chrétienne. ${ }^{11}$

Il s'agissait dans cette clause de maintenir l'obligation civile existante, et Vaudreuil demandait ni plus ni moins que le gouvernement anglais joue le rôle de bras séculier de l'Église catholique, tout comme le gouvernement français l'avait fait. Mais si ce rôle était familier et naturel à une administration française et catholique, il n'en était pas ainsi pour un gouvernement britannique et protestant, et Amherst répondit à la demande du gouverneur canadien: "L'Obligation de payer la Dixme aux Prêtres dependra de la Volonté du Roy. ${ }^{12}$

Cette réponse du général anglais ne modifiait en rien, bien entendu, la loi ecclésiastique de la dîme, et les curés pouvaient continuer à percevoir leur dû; mais la protection de leur droit par l'autorité civile était suspendue et le recours en justice n'était plus possible. Quant aux habitants, des motifs de religion, ou la crainte de sanctions ecclésiastiques, i.e. l'excommunication, ${ }^{13}$ pouvaient les tenir dans leur devoir, mais ils n'avaient à craindre aucune contrainte de l'autorité civile, ni aucune amende imposée par une cour de justice.

C'est bien le sens que Carleton donne à ce refus lorsqu'il écrit, en 1769:

... Donc l'établissement légal de cette religion avec

le droit formel de contraindre le peuple à payer les

11 Traité de capitulation de Montréal, 8 septembre 1760, art. 27, dans Doc. const., I: $13 \mathrm{~s}$.

12 Loc. cit.

13 Cette sanction de l'excommunication existait depuis très longtemps dans le droit canonique; ici, au Canada, on la voit rappelée pour la première fois dans une ordonnance de Mgr de Saint-Vallier, en date du 9 novembre 1690. Cf MEQ, I: 271 . 
dîmes, non comme une contribution volontaire, mais comme une redevance reconnue par la loi, est refusé aux dits habitants jusqu'à ce qu'il plaise à Votre Majesté d'en ordonner autrement, ce que Votre Majesté n'a pas encore jugé à propos de faire. En vertu de ce refus, toutes les parties des lois et coutumes canadiennes concernant le paiement des dîmes et autres redevances inhérentes à l'Eglise sont ou abolies ou suspendues. ${ }^{14}$

Mgr Briand lui-même, dans une lettre aux habitants de Kaskakias, le 7 août 1767 , après leur avoir reproché leurs défauts, ajoute ceci qui reflète bien la situation: "Je ne puis comme on le pouvait autrefois faire une sainte violence aux transgresseurs en m'adressant aux puissances séculières pour les forcer à rentrer dans leur devoir. $\gg 15$

Le traité de Paris, qui faisait passer définitivement le $\mathrm{Ca}$ nada sous la domination britannique, fut signé le 10 février $1763,{ }^{16}$ mais ce document n'apportait rien de nouveau sur la question des dîmes; il en fut ainsi de la proclamation royale du 7 octobre $1763^{17}$ qui n'en dit pas un mot. L'état d'incertitude créé par la conquête devait se prolonger jusqu'à l'Acte de Québec en 1774.

Nous avons vu la situation juridique de la dîme durant cette période qui va de 1760 à $1775,,^{18}$ mais on peut se demander quelle fut la situation de fait, ou, si l'on veut, quel fut l'effet de la situation juridique que nous avons décrite sur l'attitude des Canadiens. En clair, la question qui se pose est la suivante: malgré le refus britannique de reconnaître civilement le droit des curés à

14 Brouillon d'un rapport préparé par l'honorable gouverneur en chef et le Conseil de la Province de Québec, pour être présenté à sa Très-Excellente Majesté le Roi en son Conseil, au sujet des lois et de l'administration de la justice de cette Province, dans Doc. const., I: 316s.

15 Lettre pastorale aux habitants de Kaskakias, 7 août 1767, dans MEQ, 2: 205.

16 Traité de Paris, 10 février 1763, dans Doc. const., 1: 83-94.

17 Proclamation royale, 7 octobre 1763 , ibid., 136-141.

18 C'est le $1^{\text {er }}$ mai 1775 que l'Acte de Québec devait prendre force de loi. Cf Acte de Québec, 1774, ibid., 554. 
la dîme, les habitants ont-ils continué de payer cette redevance comme sous le régime français?

Pour répondre à cette question, la source d'information la plus adéquate serait sans doute les livres de comptes des curés; en comparant les entrées des dîmes avant 1760 avec celles qui ont suivi la conquête, on pourrait, en constatant la stabilité ou la diminution des revenus, toutes choses égales d'ailleurs, conclure à un maintien ou à un changement de l'attitude des Canadiens en ce domaine. Mais, justement, ces comptes de dîmes étaient et sont encore des documents strictement privés et personnels aux curés, qui n'avaient pas à être versés aux archives de fabriques, et on n'en trouve aujourd'hui à peu près aucune trace.

À défaut de cette source de première main, nous avons pour nous renseigner, d'une part, des témoignages d'officiers anglais, et d'autre part, des documents épiscopaux, la correspondance de Mgr Briand, les mandements, lettres pastorales et circulaires de l'évêque, de même que le journal de ses visites pastorales.

\section{Témoignages des officiers anglais}

Chronologiquement, le premier témoignage que nous ayons d'un officier anglais, c'est celui de Murray, dans son rapport sur le gouvernement de Québec. En 1761, Lord Égremont avait demandé dans une lettre à Sir J. Amherst, qu'on envoie le plus tôt possible à Londres un rapport complet concernant les gouvernements de Québec, Montréal et Trois-Rivières. ${ }^{19}$

Le rapport de Murray est daté du 5 juin $1762 .{ }^{20}$ En plus de statistiques très détaillées, ce rapport fournit des jugements personnels du gouverneur sur la population, la religion, la société, la noblesse, le clergé. Au sujet du clergé, le général note l’observation suivante:

L'influence du clergé a toujours été et est encore très grande; tout de même, depuis le peu de temps que nous sommes ici, nous avons constaté un change-

19 Doc. const., I: 29, note 1.

20 Rapport du Général Murray concernant le gouvernement de Québec au Canada, daté du 5 juin 1762, dans Doc. const., I: 29-66. 
ment: la population ne se soumet pas aussi facilement au joug, et tous les jours les termes de la capitulation leur fournissent quelque raison de contester les dîmes aux curés. ${ }^{21}$

Que vaut ce témoignage de Murray? Les jugements de ce gouverneur nous apparaissent souvent entachés de préjugés, mais lorsqu'il mentionne des faits, même si ces faits n'ont d'autre preuve que son témoignage, il est plus difficile de les récuser. Deux explications nous paraissent plausibles, la première qui accepte le témoignage de Murray, la seconde qui restreint ce témoignage.

Il est fort possible que, dès après la conquête, plusieurs Canadiens aient cru que l'article 27 de la capitulation de Montréal ${ }^{22}$ les relevait dorénavant de l'obligation de payer la dîme; habitués qu'ils étaient à voir sanctionner par une loi civile leurs obligations religieuses, ils pouvaient facilement s'imaginer, des interprètes intéressés de la loi aidant, que c'était en vertu de la seule loi civile qu'ils étaient tenus à ces obligations, et que cette loi ne protégeant plus le droit des curés, elle abrogeait aussi le devoir des paroissiens.

Pour appuyer cette hypothèse, nous pouvons citer un cas presque semblable, mais qui se produira cent ans plus tard, en 1868. Il s'agit dans ce cas de la capitation, redevance destinée à remplacer la dîme, et qui n'a jamais eu la reconnaissance civile; on l'avait imposée depuis quelque temps dans plusieurs diocèses de la Province de Québec, et on éprouvait, semble-t-il, des difficultés à la faire payer. Cette situation donna lieu à un décret $\mathrm{du} I \mathrm{IV}^{\mathrm{èm}}$ concile provincial de Québec:

Comme il s'est glissé dans l'esprit de plusieurs. l'erreur de croire que les dîmes et les autres contributions qui se paient à l'Eglise [...] ne se paient qu'en vertu de la loi civile, et que l'obligation de les acquitter ne vient d'aucune autre source; $[\ldots]$ nous jugeons opportun de déclarer $[\ldots]$, que cette obligation dérive et se tire spécialement de la loi ou des lois que l'Eglise a faites, et peut et pourra faire

21 Ibid., I: 64.

22 Traité de capitulation de Montréal, art. 27, ibid., I: 13s. 
indépendamment et même en l'absence de toute loi civile; $[\ldots]^{23}$

Il n'est pas impossible qu'une réaction semblable se soit produite entre 1760 et 1762 . De plus, de mauvais conseillers, fauteurs de troubles, pouvaient fort bien tenter de dissuader les habitants de payer leurs redevances aux curés, en interprétant pour eux l'article 27 de la capitulation; c'est d'ailleurs l'argument invoqué dans une pétition adressée au roi en 1765 , et où on réclame, parmi d'autres choses, la reconnaissance de la dîme:

Comme en conservant leurs prêtres ils doivent s'occuper de leur subsistance et que des habitants des campagnes, mal conseillés, pourraient refuser de leur payer la dixme, comme cela s'est toujours pratiqué dans cette province; ils supplient votre Excellence $[\ldots] .{ }^{24}$

Toute cette explication que nous avons échafaudée reste à toutes fins pratiques une hypothèse non vérifiée, tout au plus vraisemblable. Nous préférons de beaucoup la seconde explication, qui peut se résumer ainsi: Murray a raison quand il affirme que des Canadiens refusent de payer leurs dîmes, mais l'explication qu'il donne de cette attitude, l'article 27 de la capitulation, nous apparaît fausse.

Il faut bien se rappeler d'abord que le rapport de Murray est de 1762, et qu'il ne porte par conséquent que sur les deux premières années du régime militaire. Or, on sait toute la misère et l'extrême pauvreté qui étaient résultées de la guerre: abandon presque total des terres durant la guerre, dévastations opérées par les armées anglaises, mauvaises récoltes des dernières années, misères dont on ne faisait que commencer à se relever en $1762 .{ }^{25}$

${ }^{23}$ Décret XVI du IVàme Concile provincial de Québec, 1868, dans Concilia Provincix Quebecensis I, II, III, IV, 1870, 210s. Traduction française de ce décret dans Mandements de Mgr Langevin, $1^{\text {er }}$ évêque de Rimouski, vol. 2, document No 29: 3 .

24 Observations que les nouveaux sujets de Sa Majesté prennent la liberté de mettre sous les yeux de Son Excellence l'Honorable James Murray, le suppliant de vouloir bien, en leur continuant ses bontés à cette province, les présenter de leur part à leur gracieux souverain et les appuyer de sa protection. AAQ, Gouvernement, V, 25: 3.

25 Lionel Groulx, Lendemains de conquête, 21-28. 
Dans ces conditions, Murray a peut-être raison d'affirmer que des habitants ont refusé de payer la dîme, mais il semble qu'il se soit mépris sur leurs raisons d'agir ainsi. Dépourvus de tout, réduits à une très grande misère, les habitants ne pouvaient payer leurs dûs, et ce ne sont pas les dispositions du traité de capitulation qui les ont incités à ce refus.

Ce qui paraît le plus étonnant, c'est que Murray n'ignorait rien de la situation précaire des Canadiens: c'est à sa demande que M. Briand, en 1761, prescrivait à ses curés d'envoyer au gouverneur la liste des familles de leurs paroisses qui étaient dans le besoin. ${ }^{26}$

On trouve d'ailleurs un exemple de cette situation des paroissiens à l'égard de leurs curés, dans une lettre du curé de PointeLévy, M. Youville-Dufrost, adressée à M. Briand le 4 janvier $1762 ; 27$ le document tout entier est intéressant, mais nous en avons retenu quelques passages caractéristiques. $\mathrm{Au}$ sujet de fondations de messes, le curé de Pointe-Lévy demande s'il doit les acquitter: «Suis-je obligé d'acquitter ces fondations, étant aujourd'hui pauvre comme un rat d'église et ne m'attendant qu'à une dîme des plus minces ? » et plus loin le curé Dufrost ajoute: «Mes paroissiens doivent aussi la dîme de $1760 \ldots$... Il mentionne même le cas du récollet Daniel, son prédécesseur à la cure de Pointe-Lévy, «qui, ayant exposé à M. Murray que les habitants étaient hors d'état de le payer, M. Murray en conséquence lui avait donné la ration pendant longtemps ».

Comme on le voit, la misère et la pauvreté peuvent à elles seules expliquer, bien mieux que les termes de la capitulation, que la dîme, dans certains cas, n'ait pas été payée durant ces deux années.

Que conclure, donc, de l'affirmation de Murray ? Rien de très certain, rien en tout cas qui nous permette de juger l'époque, puisque son témoignage ne porte que sur les deux premières

${ }^{26}$ Lettre circulaire de M. Briand, vicaire général, demandant d'envoyer au gouverneur la liste des familles pauvres, janvier 1761, dans MEQ, 2: $149 \mathrm{~s}$.

27 M. Youville-Dufrost, curé de Pointe-Lévy, à M. Briand, vicaire général, le 4 janv. 1762. AAQ, St-Joseph de Lévis, I: 9 (4 pages). 
années du régime militaire, années de misère et de pauvreté s'il en fut. Le témoignage de Murray reste toutefois vraisemblable, et peut, comme nous l'avons montré, trouver une explication; et si, justement, nous avons tenté de l'expliquer, c'est que, parmi tous les officiers anglais dont nous avons le témoignage à ce sujet, Murray est le seul qui affirme que les Canadiens ont refusé, en se basant sur le traité de 1760 , de payer la dîme... ce qui justifie de notre part une sérieuse réserve.

Les autres témoignages directs que nous avons sur la question nous sont parvenus grâce à Sir Henry Cavendish,28 député britannique qui siégeait au parlement de Londres en 1774, et dont les notes sur le débat qui a précédé l'adoption de l'Acte de Québec, ont été publiées en 1839. ${ }^{29}$ Au cours de ce débat, pour éclairer davantage les députés sur les affaires du Canada, on fit comparaître, devant la Chambre formée en comité, les personnages qui étaient le plus en mesure de les renseigner. C'est ainsi que Carleton, Masères, Wedderburn, Marriott, Hey, furent appelés à témoigner devant les membres du parlement.

Carleton était alors gouverneur général du Canada, mais depuis 1770, il était à Londres où il travaillait à la préparation de l'Acte de Québec; appelé à témoigner devant la chambre le 2 juin 1774, on lui posa la question suivante au sujet de la dîme:

Have the clergy in Canada since the peace enjoyed and received the tithes and parochial dues?

Et voici la réponse du gouverneur à cette question:

They have received the tithes and parochial dues as formerly; they may be some who have not, but very few; as few as those gentlemen who receive their rents; they are as well paid as the rents. ${ }^{30}$

${ }^{28}$ Sir Henry Cavendish, Debates of the House of Commons in the year 1774, on the bill for making more effectual provision for the government of the Province of Quebec, publié par J. Wright.

29 Ces débats ne sont rapportés dans aucune des publications de l'époque, à cause de la loi qui interdisait la présence des étrangers aux séances de la Chambre et la publication des discours des députés. On ne connaissait, avant la publication des notes prises au cours des séances par Cavendish, que les très grandes lignes des débats parlementaires autour du bill de Québec. Cf Cavendish, op. cit., Editor's Preface, 3.

30 Cavendish, op. cit., 103. 
Masères, qui avait été procureur général de la province de Québec de 1766 à $1773,{ }^{31}$ fut appelé à son tour pour donner son opinion sur les diverses clauses de l'Acte de Québec. Après avoir rappelé la réponse du général Amherst concernant la dîme, lors de la capitulation de Montréal, ${ }^{32}$ Masères affirme que cette réponse a toujours été considérée comme «a positive dispensing with the obligation ${ }^{33}$ et il ajoute ceci: "It has often happened that they (the Canadians) have not paid tithe; much oftener that they did, from their regard to their religion. »

Carleton est formel dans son affirmation: «les Canadiens ont payé la dîme comme avant... aussi bien que les rentes», et il n'apparaît pas que la perception des rentes ait posé des problèmes après la conquête. Masères, pour sa part, semble restreindre un peu le témoignage de Carleton: «souvent il est arrivé qu'ils n'ont pas payé, mais beaucoup plus souvent ils ont payé », et il prend soin d'ajouter qu'ils l'ont fait pour un motif de religion. On peut dire qu'à peu de choses près, les deux témoignages concordent pour affirmer que les Canadiens ont continué à payer la dîme, malgré l'absence d'une législation civile pour les $\mathrm{y}$ forcer.

Un autre personnage mérite aussi qu'on s'arrête à son témoignage, le solliciteur-général Alex. Wedderburn. ${ }^{34}$ En 1772, ce conseiller-juriste de la Couronne avait eu à préparer un rapport concernant les affaires du Canada, et à présenter au «Board of Trade» ses recommandations concernant l'Acte de Québec. ${ }^{35}$ Nul doute que ce travail lui avait permis de connaître assez bien la situation du pays, et qu'il avait cherché à obtenir des informations de première main pour le conduire à bien; aussi, même si ce fonctionnaire n'est jamais venu au Canada, son témoignage vaut par l'information sur laquelle il est basé. Au sujet de la

31 Ibid., 123, note 1. Masères comparut le 3 juin 1774.

32 Traité de capitulation de Montréal, art. 27, dans Doc. const., 1: 13s.

33 Cavendish, op. cit., 130.

34 Alexander Wedderburn fut solliciteur-général de Grande-Bretagne de 1771 à 1778 . Ibid., 49 .

35 Rapport du solliciteur-général Alex. Wedderburn, 6 décembre 1772, dans Doc. const., 1: 402-412. 
dîme, voici ce qu'affirme Wedderburn, à la séance du 26 mai 1774:

As the tithe stand at present, the clergy do receive them. I believe it is equally true, that they have no suits in any temporal court. The mode of enforcing payment is by excommunication. ${ }^{36}$

L'excommunication dont parle ici Wedderburn n'était pas une législation nouvelle; nous avons vu précédemment que c'était la sentence de l'Église contre ceux qui ne payaient pas leurs dîmes, et cela au moins depuis 1690 au Canada. La réflexion du solliciteur-général ne veut pas dire qu'on aurait créé cette sanction à cause de difficultés spéciales à percevoir la dîme, mais elle veut seulement souligner que c'est à ce moment la seule sanction, puisque la loi civile n'intervient plus.

Concluons sur ces témoignages des officiers anglais: ${ }^{37}$ sauf celui de Murray, qui nous paraît douteux, et qui, en tous cas, ne vaut que pour les deux premières années du régime anglais, on peut dire que les trois autres témoignages que nous avons cités, et qui proviennent de personnages compétents, concourent dans le même sens: apparemment rien n'a changé dans l'attitude des Canadiens par rapport à la dîme.

\section{Documents épiscopaux}

Examinons maintenant la correspondance de Mgr Briand avec ses curés, les mandements et lettres pastorales de l'évêque durant cette période de 1760 à $1775^{38}$ ainsi que le journal de ses visites pastorales. Cette source nous paraît être la plus importante de celles que nous possédons pour éclairer notre problème.

En effet, si, à partir de 1760, la question des dîmes, en raison du refus de l'Angleterre de les reconnaître, a posé des

36 Cavendish, op. cit., 69s.

37 Nous avons volontairement laissé de côté quelques autres témoignages donnés à la Chambre des Communes, parce qu'ils provenaient de députés qui n'avaient pas une connaissance particulière de la question, et qui ne faisaient que répéter ce que d'autres avaient dit; c'est le cas par exemple de M. S. Glynn, cf Cavendish, op. cit., 69.

38 Il s'agit évidemment des lettres de M. Briand, vicaire général jusqu'en 1766, et de Mgr Briand, évêque de Québec à partir de 1766 . 
problèmes aux autorités religieuses du pays, on doit certainement en trouver des échos dans la correspondance de l'évêque avec ses curés; si de plus ce problème a pris des proportions diocésaines - on pourrait même dire nationales -, Mgr Briand a sûrement publié quelque lettre pastorale ou mandement, pour exposer la doctrine, revendiquer les droits des curés et rappeler leurs devoirs à ses diocésains, ou du moins l'aura-t-il noté au cours de ses visites pastorales.

$\mathrm{Si}$, au contraire, les habitants ont continué « as formerly ${ }^{39}$ à payer leurs dîmes aux curés, les documents cités seront silencieux, et on aura alors un témoignage, négatif sans doute, mais probant quand même, que le paiement des dîmes n'a pas posé de problèmes durant cette période.

\section{a) Les lettres de Mgr Briand}

Les lettres de Mgr Briand constituent, aux archives de l'Archevêché de Québec, un volumineux dossier; elles sont classées, selon leur destinataire ou leur sujet, sous les rubriques suivantes: Diocèse de Québec, Évêques de Québec, Gouvernement, Copies de Lettres, etc. . . ${ }^{40}$

Or, un fait nous a frappé dans cette correspondance considérable, et il importe de le signaler tout de suite, c'est la rareté des documents relatifs à la dîme.

On rencontre quelques lettres où il est question de revenus modiques. M. Marchand, par exemple, vicaire général, prévient Mgr Briand que M. de Contrecœur doit lui écrire pour sa paroisse dont le revenu est très modique; aussi croit-il «qu'il serait nécessaire que dans ces paroisses où la dîme ne suffit pas pour la subsistance du curé, les paroissiens suppléassent par quelques fournitures extraordinaires aux suppléments qu'on leur donnait autrefois. $\gg 41$

\footnotetext{
39 Carleton devant le comité de la Chambre des Communes, Cavendish, op. cit., 103.

40 Ivanhoë Caron, Introduction à l'inventaire des Lettres de Mgr Briand. RAPQ (1929-30), 45s.

$41 \mathrm{M}$. Etienne Marchand, vicaire-général, à $\mathrm{Mgr}$ J.-O. Briand, Boucherville, 24 juillet 1766, dans RAPQ (1947-48), 101.
} 
Une plainte semblable est portée par M. François Dezery, qui, nommé en 1768 "vicaire en chef ${ }^{42}$ de la paroisse de Québec, écrit à son évêque, en mai 1769, pour demander un supplément:

[...] Il supplie Votre Grandeur de vouloir bien engager messieurs les marguillers ou les habitants de la paroisse de Québec à suppléer à la modicité des revenus de la dite paroisse et à mettre le curé en état d'en acquitter les charges $[\ldots]^{43}$

M. Dezery ajoutait à sa requête un état de ses dépenses et du produit de son bénéfice. ${ }^{44}$

Si nous avons cité ces deux cas, c'est pour bien montrer que si certains curés ont des revenus modiques, ce n'est pas la faute de leurs paroissiens, mais bien, semble-t-il, parce que le supplément payé sous le régime français par le roi ne vient plus combler une insuffisance qui a toujours existé.

Nous avons parlé, en commentant le témoignage de Murray de l'état de misère qui sévissait au pays dans les premières années du régime militaire, et qui pouvait expliquer certaines défaillances dans le paiement des dîmes; dans une lettre à l'un de ses curés, M. Briand fait écho à cette situation pénible de la colonie:

Votre paroisse est bonne, écrit-il à M. Maisonbasse, vous avez la meilleure de ce gouvernement; vous ne devés pas exiger à l'extrême dans ces temps de misère générale, et cela serait encore d'un mauvais exemple auprès de notre gouverneur qui paraît si affectionné pour les pauvres. ${ }^{45}$

Notons tout de suite que cette remontrance de M. Briand à M. Maisonbasse n'a pas de rapport direct avec la dîme, mais

${ }^{42} \mathrm{Mgr}$ Briand à M. Marchand, vicaire-général, Québec, 22 avril 1768, $\mathrm{AAQ}$, Lettres, III : 420.

43 M. François Dezery, curé d'office de Québec, à Mgr Briand, Québec, mai 1769. ANDQ, carton 9, No 5 . Après bien des tergiversations, les marguilliers accordèrent, le 20 déc. 1773, 450 livres pour le loyer du curé et un supplément de 1200 livres; cependant ce n'était plus M. Dezery qui était curé, mais M. Dosque. Ibid., carton 9, No 8.

44 Ibid., No 11.

45 M. Briand, vicaire-général, à M. Maisonbasse, curé à St-Thomas, Québec, 21 février 1761, AAQ, Lettres, III : 41. 
qu'elle porte sur un projet de construction du presbytère, pour lequel le curé de St-Thomas voulait faire payer ses paroissiens. Ce qui importe pour nous dans cette lettre, c'est que, datée de 1761, elle montre bien que, contrairement à Murray, qui témoignait de la même époque, $M$. Briand interprète les difficultés rencontrées par les curés à se faire payer tous leurs dûs, comme le résultat de la misère qui règne au pays. Et encore, malgré cette 《misère générale », ce n'est pas le problème de la dîme qui se pose au curé Maisonbasse, mais celui de la construction d'un presbytère.

Deux cas cependant, relevés dans cette correspondance de Mgr Briand, nous ont paru mériter une étude plus approfondie, parce que, dans ces deux cas, il s'agit bien du refus obstiné de certains habitants à satisfaire à leurs obligations, à St-François de Beauce et à Cap St-Ignace.

Le cas de St-François nous est connu par la correspondance échangée entre Mgr Briand et M. Verreau. Ce dernier était curé de Ste-Marie de Beauce et il desservait les paroisses de St-François et de St-Joseph. Dans une lettre qu'il adresse au curé, le 24 avril 1772, Mgr Briand parle de
deux ou trois mutins qui ont répandu qu'ils s'a- dresseraient à $\mathrm{Mr}$ Carleton lorsqu'il serait arrivé, qu'ils n'étaient pas obligés à la dixme, que vous ne la gagniés pas, que c'était vous qui ne vouliés pas aller chez eux, que leurs enfants ne sçavaient pas un mot de catéchisme, et qu'ils avaient eu peur de vous comme du diable lorsqu'une fois vous allâtes à St- François et qu'ils s'enfuirent de frayeur dans les bois. ${ }^{46}$

Ce n'était pas très flatteur pour M. le curé, mais Mgr Briand ne faisait que lui répéter les griefs qu'on avait contre lui dans sa paroisse, et qui semblent avoir été formulés dans une lettre adressée à l'évêque, mais que nous n'avons pu retrouver; en somme, ces quelques mutins refusaient de payer leur dîme, parce que, prétendaient-ils, le peu de service qu'ils recevaient de leur curé ne justifiait pas un tel paiement. Que se passait-il

${ }^{46} \mathrm{Mgr}$ Briand à M. Jean-Marie Verreau, 24 avril 1772, AAQ, StFrançois de Beauce, 1: 3 . 
exactement à St-François ? Une requête des paroissiens, conservée aux archives de l'archevêché de Québec,, ${ }^{47}$ nous éclaire un peu plus sur la situation: on y apprend qu'à la suite d'actes répréhensibles, commis par «quelques étourdis 》 de St-François, Mgr Briand avait interdit l'église, et obligé ainsi les paroissiens à se rendre à St-Joseph pour le service religieux; ceux-ci, se trouvant mal desservis, demandent à l'évêque de lever l'interdit qui pesait sur leur église, et surtout de leur donner un curé résidant, et ils ajoutent que

quoi qu'il ait été dit que les trois paroisses ne sont point en état réunies ensemble de nourrir un curé; les habitans de St-François s'efforceraient non seulement de prouver le contraire mais même de faire voir qu'une seule peut en entretenir un honorablement. ${ }^{48}$

Ce dernier texte nous prouve que les habitants de St-François n'ont pas d'objection à payer pour faire vivre un curé, bien au contraire; pour avoir un curé bien à eux, ils sont prêts à payer davantage.

L'année suivante pourtant, dans une lettre à son évêque, M. Verreau se plaint de l'attitude de ses paroissiens relativement à la dîme:

Si j'osais, je demanderais à Votre Grandeur une lettre d'avis pour mes trois paroisses touchant la dixme, il me paraît qu'il y a bien de l'abus parmi eux sur cet article. La voix du premier pasteur leur fera plus d'impression que celle du pasteur ordinaire. Il est assez général d'entendre dire qu'un habitant a de droit un arpent de terre qui ne doit pas payer dîme. En conséquence on fait de grands jardins, et là les fèves, le bled-dainde et souvent les patattes sont à couvert de tout droit. Il y en a même, à ce qu'on m'a dit, qui avancent qu'on ne doit pas la dîme du bled de semance, parce que ce serait payer deux fois. Si on s'en rapportait à eux, je crois qu'ils viendraient bientôt about d'affranchir la moitié de leurs terres, vu qu'il y en a plusieurs qui

${ }^{47}$ Requête des paroissiens de St-François, AAQ, St-François, I: 1. 48 Ibid., feuillet 2. 
sèment jusqu'à deux et trois minots de graine de lin dont on ne leur demande rien. ${ }^{49}$

Nous avons cité longuement cette lettre de 1773, qui nous paraît comporter un intérêt particulier: c'est une répétition à peu près intégrale de la querelle de 1705 , alors que les curés de Beauport et de l'Ange-Gardien avaient réclamé la dîme de toutes les cultures, et même de l'élevage, sous prétexte que les habitants diminuaient de plus en plus la culture des grains décimables. ${ }^{50}$

On peut trouver que les paroissiens du curé Verreau, de ses trois paroisses cette fois, sont un peu mesquins, mais on ne voit pas que les motifs de leurs agissements soient inspirés de l'article 27 de la capitulation de Montréal: il s'agit pour eux d'en payer le moins possible...; s'ils avaient prétendu qu'ils n'étaient pas tenus de payer parce que la loi civile ne les obligeait pas, ils auraient tout simplement refusé leur paiement.

Un autre cas, très complexe celui-là, et où la question des dîmes est intervenue à quelques reprises, c'est le cas du Cap StIgnace. Les difficultés religieuses de cette paroisse pourraient à elles seules faire le sujet d'un livre important, et en $1781 \mathrm{Mgr}$ Briand pouvait écrire dans une lettre pastorale aux habitants du Cap St-Ignace: "Il y a plus de soixante ans que le trouble règne dans cette paroisse ${ }^{51}$ On peut dire que toutes les décisions de l'administration religieuse, concernant l'église, le presbytère, le cimetière, avaient engendré des chicanes, suscitées surtout par la rivalité entre les censitaires du fief Gamache et ceux du fief Vincelotte, qui voulaient tous avoir le bon Dieu à leur porte.

En 1766, Mgr Briand menace les paroissiens de leur enlever leur curé, ${ }^{52}$ menace qu'il met à exécution en 1769 , alors qu'il

\footnotetext{
49 M. Verreau à Mgr Briand, 9 octobre 1773, AAQ, Ste-Marie, I: 4.

50 Sur toute cette affaire de 1705, voir l'étude de l'abbé Auguste Gosselin, Un épisode de l'histoire de la dîme au Canada, 1705-1707, dans MSRC, $2^{\text {ème }}$ série, vol. IX (1903) section 1: 45-63.

51 Mgr Briand, Lettre pastorale aux habitants de Cap St-Ignace, Québec, 5 septembre 1781, AEP, Cap St-Ignace, I-48. ibid., $1-20$.

$52 \mathrm{Mgr}$ Briand aux habitants de Cap St-Ignace, 23 octobre 1766, AEP,
} 
confie la desserte du Cap St-Ignace à M. Jacques Hingan, curé de L'Islet. ${ }^{53}$ Dans la correspondance de Mgr Briand avec M. Hingan, nous trouvons quatre documents où il est fait mention du refus de certains habitants de payer la dîme.

En 1769, Mgr Briand écrit au curé de L'Islet que «les habitants qui refusent de payer la dîme seront condamnés au tribunal de Dieu $\gg .{ }^{54}$ On comprend aisément que, privés depuis quelques mois de leur curé, certains paroissiens se soient fait tirer l'oreille pour payer leurs redevances à un curé qui ne résidait même pas chez eux.

Trois ans plus tard, en 1772, l'évêque manifeste son contentement au curé parce que «il s'est adressé à Mrs les Juges pour les dixmes d'Augustin Bernier (du Cap St-Ignace) »;55 et plus loin dans sa lettre, il ajoute:

Si vous aviés des témoignages reçus en règle, je prononcerais des censures contre toute cette famille de Bernier, non pas contre les domestiques, à moins qu'ils ne fussent coupables, mais contre père, mère et enfans.

Un obstiné, sans doute, que cet Augustin Bernier; rien d'étonnant d'ailleurs, car en 1749 , ce même Bernier, qui s'était permis de présider aux sépultures dans le cimetière des Gamaches, interdit l'année précédente par $\mathrm{Mgr}$ de Pontbriand, avait été frappé d'une sentence d'excommunication et d'une amende de trois cents livres. ${ }^{56}$ On ne voit pas qu'en 1772 Bernier se soit autorisé des termes de la capitulation pour refuser de payer sa dîme; sa résistance s'explique suffisamment par sa passion et

${ }^{53}$ Archives de la fabrique de L'Islet, 1: 23s. En 1786, Mgr Briand avait retardé d'un an le départ du curé, à la demande même de ce dernier (M. Charles Garault St-Onge). Cf Mgr Briand aux habitants de Cap StIgnace, 21 octobre 1768, AEP, Cap St-Ignace, 1-19.

54 Mgr Briand à M. Jacques Hingan, (1769 ?) AAQ, Lettres, III: 365.

55 Le même au même, 8 mai 1772, AEP, Cap St-Ignace, 1-40: 1r.

56 Sirois, Monographie de St-Ignace du Cap St-Ignace de 1672 à 1903, 36. L'auteur ajoute aux détails que nous avons cités: «Plus tard, en 1785, les rôles changèrent, ce ministre improvisé eut besoin à son tour de quelqu'un pour présider à sa sépulture. Heureusement alors que les cérémonies se firent dans l'ordre, puisqu'on lit à l'acte d'inhumation d'Augustin Bernier, qu'il mourut «muni de tous les sacrements de l'Eglise », âgé d'environ 80 ans 》. Loc. cit. 
par l'esprit général de chicane qui régnait dans la paroisse depuis si longtemps.

Un fait, dans cette affaire, a quelque raison de nous étonner: contrairement à l'article 27 de la capitulation de Montréal, qui refuse de reconnaître la dîme, le curé de l'Islet s'est adressé aux juges pour se faire payer la dîme d'Augustin Bernier. ${ }^{57}$ Cela semble indiquer que dans bien des domaines, on en était revenu, en 1772, à la coutume en vigueur avant la conquête. Carleton avait déjà signalé un certain nombre de ces cas où la loi civile française était demeurée en force, ${ }^{58}$ et le juge en chef Hey, lors de l'étude du bill de Québec en 1774, affirmera devant le parlement de Londres qu'il s'est cru obligé de juger les causes en appel en tenant compte des lois françaises, parce qu'on basait aussi sur elles les jugements de la Cour du Banc du Roi. ${ }^{59}$ Dans ces conditions, on imagine difficilement comment les habitants auraient pu invoquer un article du traité de capitulation dont les juges eux-mêmes ne tenaient pas compte.

La même année 1772, Mgr Briand envoie aux habitants de Cap St-Ignace une nouvelle lettre pastorale dans laquelle il menace d'excommunication majeure «tous ceux qui dans la paroisse de Cap St-Ignace n'ont point satisfait au devoir paschal ni payé les dixmes, ont donné conseil de ne les point payer ou y ont applaudi ; $^{60}$ dans la même lettre, l'évêque parle de ces personnes qui méprisent « nos conseils et nos avis, les ordonnances de l'Église notre mère et les jugements des personnes que notre

57 Nous n'avons pu trouver de documents judiciaires sur cette affaire, mais nous avons une lettre du curé Hingan, dans laquelle il relate cette intervention des juges: « (...) je me serais déterminé à le faire assigner par devant $M^{\text {rs }}$ les Juges de paix, et ils l'auraient condamné à me payer 12 piastres pour deux années de dîmes ce qui ne ferait pas la moitié de sa dîme des deux années. 》M. Hingan à Mgr Briand, 12 avril 1772, AEP, Cap St-Ignace, $1-40: 1 \mathrm{v}$.

58 Brouillon d'un rapport préparé par l'honorable Gouverneur en chef et le Conseil de la Province de Québec, pour être présenté à Sa Très Excellente Majesté le roi en son Conseil, au sujet des lois et de l'administration de la justice en cette province. Dans Doc. const., 1: 304-350, spécialement 322-326.

59 Examination of Chief justice William Hey, 3 juin 1774, dans Cavendish, op. cit., 151.

$60 \mathrm{Mgr}$ Briand aux habitants de Cap St-Ignace, 26 juin 1772, AEP, Cap St-Ignace, 1-37: 2r. 
gracieux souverain nous a données pour rendre la justice et maintenir les hommes dans l'ordre et réprimer les mauvais sujets ${ }^{61}$ S'il diffère davantage de punir les coupables, Mgr Briand craint que «les faibles n'en soient scandalisés: ils pourraient penser (...) que l'autorité ni la civile ni l'ecclésiastique n'ont plus de force $\gg{ }^{62}$

Encore dans ce document, Mgr Briand semble lier étroitement la loi ecclésiastique et la loi civile, ce qui était de nature à rendre vains les appels présumés des habitants à l'article $27 \mathrm{du}$ traité de capitulation, article dont, nous le répétons, on ne paraît pas avoir fait beaucoup de cas: le fait suivant confirme d'ailleurs cette hypothèse.

En 1774, Mgr Briand avise le curé Hingan qu'il ne peut exempter le sieur Vincelotte de payer les dîmes, et qu'il ne peut lui donner l'absolution s'il refuse de les payer:

c'est à l'Eglise et vous n'avez pas droit de les lui remettre ni de lui donner l'absolution sans qu'il les paye; l'église vous les a donné et l'état. ${ }^{63}$

Ce dernier mot, sous la plume de l'évêque de Québec, confirme encore une fois le fait que la législation civile du régime français était constamment invoquée même avant l'Acte de Québec; il est vrai que cette dernière lettre précède de quelques mois seulement l'Acte de Québec, et que peut-être Mgr Briand avait eu de bonnes nouvelles du gouverneur Carleton, dont le retour à Québec ne devait s'effectuer que le 18 septembre de cette année $1774 .^{64}$

Chicanes de Canadiens têtus et obstinés, voilà bien comment on peut qualifier ces disputes de Cap St-Ignace... qui ne se terminent pas d'ailleurs en $1774 .^{65}$ Ce qui nous intéresse surtout ici, c'est de constater que ces troubles avaient commencé bien avant

61 Ibid., 1 r.

62 Ibid., $2 \mathrm{r}$.

$63 \mathrm{Mgr}$ Briand à M. Jacques Hingan, curé de L'Islet, 29 mars 1774, $\mathrm{AAQ}$, Lettres, IV : 487.

${ }^{64}$ La Gazette de Québec, No 506, jeudi, 22 septembre 1774.

$65 \mathrm{En} \mathrm{1781,} \mathrm{Mgr} \mathrm{Briand} \mathrm{envoie} \mathrm{encore} \mathrm{une} \mathrm{lettre} \mathrm{pastorale} \mathrm{aux} \mathrm{habi-}$ tants de Cap St-Ignace, AEP, Cap St-Ignace, 1-48. A la suite de cette lettre, l'église fut interdite ( ${ }^{\text {er }}$ octobre 1781 ) et le demeura jusqu'au 5 juillet 1782 . Sirois, op. cit., 40. 
la capitulation du pays, et que les motifs en étaient tout autres que les termes de cette capitulation. On peut supposer, fort justement, que les événements de Cap St-Ignace auraient été les mêmes sous une administration qui serait restée française, et que Augustin Bernier, pour un, aurait tout aussi bien résisté à l'intendant français qu'au juge anglais.

Cet épisode de la vie de la paroisse de Cap St-Ignace est peut-être le plus significatif de ceux que nous avons cités, car il nous apporte une preuve du peu de changements apportés, dans la pratique de l'administration civile et judiciaire, par la capitulation de 1760 et la proclamation royale de 1763 . Les Canadiens n'ignoraient sans doute pas qu'ils avaient changé d'allégeance, mais dans leur vie quotidienne, ce changement de gouvernement ne semble pas avoir modifié la routine de vie du régime français.

Cet inventaire que nous avons fait de toutes les lettres où il est question de la dîme dans la correspondance de Mgr Briand nous a permis de voir que, si parfois il s'est posé des problèmes particuliers relativement à cette question, et nous avons pu noter qu'ils sont très peu nombreux, ces problèmes n'ont pas été suscités ni aggravés par les restrictions du traité de capitulation de 1760.

Nous nous garderons bien de conclure tout de suite de façon définitive, car la conquête a pu poser des problèmes de dîmes que l'évêque de Québec n'a pas voulu régler dans des lettres particulières; nous pensons, par exemple, que si la question de la dîme a posé un problème général, Mgr Briand a dû intervenir par des décrets ou mandements, et donner ainsi à son intervention une portée générale. C'est pourquoi nous devons maintenant examiner les documents officiels émanés de Mgr Briand, vicaire-général ou évêque de Québec.

\section{b) Lettres pastorales et mandements de Mgr Briand.}

A plusieurs reprises, surtout au cours de son épiscopat, Mgr Briand a adressé des lettres à ses diocésains, particulièrement à l'occasion de deux jubilés qui furent célébrés pendant son règne, 
en $1767^{66}$ et en $1771,{ }^{67}$ et à l'occasion aussi de ses visites pastorales. ${ }^{68}$ Chacune de ces lettres rappelle aux fidèles leurs devoirs, et les exhorte à fuir le péché et surtout à corriger les abus qui sont particulièrement à l'ordre du jour. Il faut ajouter à ces lettres deux autres mandements où l'évêque tient le même langage à ses ouailles, l'un en 1771, à l'occasion de la nomination d'un coadjuteur, ${ }^{69}$ et l'autre, en 1774 , pour célébrer son entrée dans sa cathédrale. ${ }^{70}$

En tout nous avons là treize documents où l'évêque fustige tour à tour l'ivrognerie, les manquements au jeûne et à l'abstinence, les fautes contre la sanctification du dimanche, les injustices, les impuretés de toutes sortes, la lecture des livres impies, la désobéissance aux pasteurs. L'ivrognerie est le vice qu'on trouve le plus souvent flétri sous la plume de Mgr Briand, puisqu'elle apparaît dans neuf des treize documents que nous avons examinés : dans la lettre à l'occasion de son entrée en sa cathédrale, en particulier, il déplore que ce défaut se répande surtout chez les femmes et chez les pauvres. ${ }^{71}$

Malgré l'abondance de ces documents, et surtout malgré leur caractère d'un rappel des commandements les plus négligés, pas une seule fois il n'est question du commandement de l'Église relatif aux dîmes; le mot dîme lui-même ne s'y rencontre pas. Il

\footnotetext{
$\overrightarrow{66}$ Mandement pour le jubilé accordé par N.S.P. le Pape Clément XIII, 26 janvier 1767, dans MEQ, II: 191-199. Ce jubilé avait été décrété par le pape en 1758, mais à cause des troubles de la guerre, les Canadiens n'avaient pu y participer, et $\mathrm{Mgr}$ Briand obtint de célébrer ce jubilé en $1767 \mathrm{au}$ Canada. Ibid., 191.

67 Mandement pour le jubilé universel accordé par Clément XIV, 28 janv. 1771, ibid., 218-22. Mandement du même jubilé pour la ville de Québec, 5 mars 1771, ibid., 225-227.

68 Les mandements pour les visites pastorales portent les dates suivantes: 22 mai 1767, ibid., 200-204; 1768, ibid., 211-212; $1^{\text {er }}$ juin 1771, ibid., $238-240$; 1772, ibid., 244; 1772, ibid., $245 ; 25$ fév. 1773, ibid., 245-246; 1773, ibid., 247; 22 mai 1775, ibid., 259-264.

69 Mandement à l'occasion de l'élection d'un coadjuteur, ibid., 241-243. Ce mandement, tel qu'il existe dans les archives de l'archevêché de Québec, est écrit de la main de Mgr Briand, mais ne porte ni date ni signature; il a dû être fait en 1771 (ibid., p. 243, note).

${ }^{70}$ Mandement de $\mathrm{Mgr}$ l'évêque de Québec à l'occasion de son entrée dans sa cathédrale le jour anniversaire de sa consécration, et la huitième année révolue de son épiscopat, 10 mars 1774, ibid., 247-252.

71 Ibid., 251.
} 
semble donc qu'il n'y ait pas eu d'abus à réprimer de ce côté, et que les fidèles n'ont pas oublié le sens, religieux d'abord, de la loi de la dîme.

Nous ne voulons pas conclure, de l'examen de ces documents, que Mgr Briand n'a jamais rappelé à ses ouailles leur devoir de soutenir leurs curés par le paiement des dîmes; Mgr Briand luimême nous affirme le contraire: dans une lettre à $\mathrm{M}$. Pierre Gibault, missionnaire aux Illinois, l'évêque rappelle les grands principes de l'Église relativement à la dîme:

(...) instruisés-les au sujet des dixmes, elles sont dues à Dieu et n'eussent-ils point de Missionnaires ils les devraient à Dieu comme autheur de la nature et de tous biens, le droit est naturel et aussi ancien que le monde $(\ldots) .^{72}$

Plus loin dans cette lettre, Mgr Briand ajoute:

Voilà Monsieur ce que l'on doit penser des dixmes; voilà la doctrine que j'ai enseignée dans mes visites plus détaillée, mais je vous donne les principes, et vous les développerés à ces pauvres peuples ignorans, qui je suis seur, n'ont pas péché par malice (...)

Mgr Briand a donc instruit ses peuples de leur devoir concernant les dîmes, au cours de ses visites pastorales; rien que de très naturel cependant, car c'est l'habitude des évêques, pendant ces visites, de faire une instruction portant sur tous les devoirs du chrétien, et il n'était pas nécessaire que des problèmes particuliers se soient posés à cet égard pour que l'évêque parle de la dîme en ces occasions. On n'a d'ailleurs aucun indice qu'il en ait parlé en vue de réprimer un abus généralisé.

c) Journal des visites pastorales de Mgr Briand.

Il nous reste à examiner un autre document, d'un caractère particulier celui-là, et très révélateur en ce qui concerne la question de la dîme: il s'agit du journal que Mgr Briand a tenu pendant ses tournées pastorales et où on trouve une foule de détails intéressants sur chacune des paroisses qu'il a visitées.

${ }^{72} \mathrm{Mgr}$ Briand à M. Pierre Gibault, missionnaire aux Illinois, 16 août 1770, AAQ, Lettres, IV: 145s. 
Ce document, écrit pour la plus grande partie de la main même de l'évêque, nous permet de visiter deux fois toutes les paroisses du diocèse, une première fois de 1767 à $1769^{73}$ et une seconde fois de 1771 à $1773 .{ }^{74} \mathrm{Mgr}$ Briand y a noté le nombre de communiants, la quantité de dîmes perçues par le curé, l'état des registres et des comptes, etc....; les remarques que l'évêque a faites à chacun des curés, concernant les comptes, les ornements, la propreté de l'église, les réparations à faire, et même l'âge de la servante, il les a consignées dans ce cahier, de même que les principaux problèmes propres à chaque paroisse, comme le choix d'un emplacement pour l'église ou le cimetière, l'établissement d'une répartition, l'attitude des marguilliers, etc....

Nous avons parcouru ce cahier où sont notés des renseignements précieux sur 87 paroisses ou dessertes, visitées de 1767 à 1769 et de 1771 à 1773 . Or pas une seule fois il n'y est question de la dîme, sauf bien entendu pour la quantité recueillie, qui y est scrupuleusement inscrite pour chaque paroisse visitée; pas une seule fois la dîme n'y est mentionnée comme un problème, et cela, dans aucune des 87 paroisses.

Ce fait nous semble fort révélateur, et l'argument qu'on peut en tirer apparaît plus probant encore que celui qui nous est fourni par les documents épiscopaux officiels. Car à supposer que Mgr Briand aurait eu des raisons de ne pas manifester publiquement une situation difficile concernant la dîme, on ne voit pas de raisons qui l'auraient empêché de noter ces difficultés dans son carnet de voyages, qu'on peut considérer comme un document strictement privé et personnel.

L'ensemble des documents épiscopaux que nous avons étudiés révèle, à n'en pas douter, une situation tout à fait normale en ce qui concerne la dîme. Il eut été hasardeux, pour ne pas dire ridicule, de se fier aux seules lettres, aux seuls mandements, ou uniquement au journal des visites pastorales de Mgr Briand, pour

73 Ordonnances de Mgr Briand pendant le cours de ses visites commencées le 22 juin 1767 et continuées le 31 mai 1768 , AAQ, cartable Diocèse de Québec, II : 15 . La visite de $1767-68$ compte 11 feuillets (22 pages) après quoi commence le journal de la visite de 1771-72, qui compte 5 feuillets (10 pages).

${ }_{74}$ Suite de la visite de 1772-73, ibid., 17. 
en tirer une conclusion; mais l'ensemble de ces sources, croyonsnous, constitue une preuve suffisante pour nous permettre de conclure avec certitude.

Nous n'ignorons pas que les lettres de Mgr Briand qui ont été conservées, ne constituent peut-être pas toute la correspondance de l'évêque de Québec, et que probablement plusieurs de ses lettres ont été perdues; ce fait pourrait, semble-t-il, affaiblir notre conclusion, car nous sommes en présence d'un argument qui n'a une valeur de certitude que dans la mesure où tous les documents ont pu être consultés et vérifiés.

Nous croyons cependant que l'absence de quelques lettres ne peut infirmer notre conclusion, parce que cette absence est suffisamment compensée par les documents officiels, mandements et lettres pastorales, et par cet autre document privé que nous avons cité, le journal des visites. Or cet ensemble forme un dossier impressionnant, et la concordance des conclusions fournies par chacune des pièces de ce dossier éclaire, à notre avis, l'exacte situation de la dîme entre 1760 et 1775.

\section{CONCLUSION}

Le titre même de cette étude posait une question: nous croyons pouvoir affirmer que nous avons répondu à la question posée.

Les deux groupes de témoins que nous avons interrogés ont donné des réponses identiques, les premiers par leurs affirmations, les seconds par leur silence; il nous semble toutefois que c'est le silence des seconds qui peut davantage nous amener à une conclusion à peu près définitive.

Nous ne négligeons pas pour autant les témoignages des officiers anglais: sauf Murray, dont le témoignage, nous l'avons vu, appelle de sérieuses réserves, tous les autres ont affirmé que les Canadiens, pour des motifs de religion, ont continué à payer leurs dîmes comme auparavant, entre 1760 et 1775 . Cependant ces seuls témoignages avaient besoin d'être prouvés, et c'est le silence des documents épiscopaux sur le sujet qui vient confirmer les témoignages donnés par les fonctionnaires britanniques. 
L'historien Burt a semblé voir dans la gratitude manifestée par le clergé canadien envers le gouvernement anglais pour l'Acte de Québec, un indice des difficultés que le même clergé aurait rencontrées au sujet de la perception des dîmes depuis 1760 ; c'est là, pour Burt, le seul témoignage indirect que nous ayons sur cette question de la dîme, et il admet qu'il est fort douteux, car le clergé avait bien d'autres raisons de se réjouir de l'Acte de Québec, qui semblait régler en sa faveur plusieurs questions importantes.75 Nous croyons avoir apporté notre modeste contribution pour éclairer davantage la question, et annuler ainsi la valeur de ce témoignage douteux.

Il aurait été intéressant d'étudier la genèse de la clause des dîmes dans l'Acte de Québec, ainsi que les délibérations qui ont précédé son adoption à la Chambre des Communes de Londres, et les conséquences de cette clause ; mais cet aspect légal de l'histoire de la dîme n'entrait pas dans notre propos.

Retenons seulement que l'Acte de Québec rétablissait par cette clause toute la législation du régime français concernant la dîme, en restreignant toutefois les droits du clergé catholique aux seules dîmes de ses ressortissants. ${ }^{76}$

L'Acte de Québec comblait une lacune légale, en reconnaissant le droit de subsistance aux ministres d'une religion reconnue elle-même par la loi, et mettait fin ainsi à l'incertitude et à l'instabilité qui régnaient depuis 1760 ; mais il n'avait aucunement à corriger une situation de fait, puisque, nous croyons l'avoir démontré, malgré l'attitude des vainqueurs de 1760 , les Canadiens avaient continué de payer la dîme, et que même, au moins dans un cas, les tribunaux civils étaient intervenus pour faire appliquer la loi de la dîme.

Georges-Étienne Proulx, ptre, Licencié ès lettres (Histoire) de l'Université Laval, Professeur d'histoire au Collège de Lévis (Québec, Canada).

75 Burt, The Old Province of Quebec, 188s. Il ne faut pas oublier que les instructions à Carleton, en 1775 , restreignaient singulièrement la portée des faveurs accordées par l'acte de $\mathbf{1 7 7 4}$, même en ce qui concerne la dîme. Cf Instructions à Carleton, 3 janvier 1775, dans Doc. const., 1: 588.

${ }^{76}$ L'Acte de Québec, 1774, dans Doc. const., 1: 554. 


\section{BIBLIOGRAPHIE}

\section{I - Sources manuscrites}

En raison du sujet de notre travail, qui relève avant tout de la législation ecclésiastique, nous avons surtout utilisé des documents d'archives religieuses, principalement à l'Archevêché de Québec. Pour quelques questions de détail, nous avons eu recours aussi aux archives de l'évêché de Ste-Anne de la Pocatière et à celles de la paroisse NotreDame de Québec.

a) Archives de l'Archevêché de Québec.

Lettres, III et IV (copies des lettres des évêques).

Diocèse de Québec, II.

Evêques de Québec, I.

Gouvernement, V.

St-François de Beauce, I.

Ste-Marie de Beauce, I.

St-Joseph de Lévis, I.

b) Archives de l'Evêché de Ste-Anne de la Pocatière. cartable Cap St-Ignace, I.

c) Archives de la paroisse Notre-Dame de Québec. carton 9.

II - Sources imprimées

a) Documents civils.

Edits, Ordonnances royaux, déclarations et arrêts du Roi concernant le Canada. Québec, E.-R. Fréchette, 1854-1856. 3 vol.: 648; 650;776p. $25 \mathrm{~cm}$.

Documents relatifs à l'histoire constitutionnelle $d u C a-$ nada, 1759-1791. Choisis et édités avec notes par Adam Shortt et Arthur Doughty. Seconde édition revisée par le bureau de Publication des documents historiques. Ottawa, 1921. 2 volumes: XIX-1064p. $25 \mathrm{~cm}$.

Sir Henry Cavendish. Debates of the House of Commons in the year 1774, on the bill for making more effectual provision for the government of the Province of Quebec, drawn up from the notes of the right honourable Sir Henry Cavendish, member for Lostwithiel, now first published by J. Wright, editor of the parliamentary history, etc.... with a map of Canada, copied from the second edition of Mitchell's map of North America referred to in 
the debates. London, J. L. Cox and Sons, 1839. XII-303p. $23 \mathrm{~cm}$. maps.

b) Documents religieux.

Concilia Provincix Quebecensis I, II, III, IV in Quebecensi civitate celebrata et a sancta sede revisa et recognita. Quebeci, apud P.-G. Delisle typographum, $1870.320 \mathrm{p} .22 \mathrm{~cm}$.

Mandements, lettres pastorales et circulaires des Evêques de Québec. Publiés par Mgr Henri Têtu et l'abbé C.-O. Gagnon. Volume deuxième. Québec, A. Côté \& cie, 1888.566 p. $22.5 \mathrm{~cm}$.

Mandements, lettres pastorales et circulaires de Mgr Jean P.F.L. Langevin, premier évêque de St-Germain de Rimouski, $2^{2 m e}$ volume, du $1^{\text {er }}$ janvier 1872 au 31 décembre 1875 . Rimouski, $1876.20 \mathrm{~cm}$. C'est une collection de documents dont chacun a sa propre pagination.

Journal des visites pastorales de 1815 et 1816 par Mgr Joseph-Octave Plessis, évêque de Québec. Publié par Mgr Henri Têtu. Québec, Imprimerie Franciscaine missionnaire, 1903 . 205-75p. $23.5 \mathrm{~cm}$.

\section{III - Inventaires}

Rapport de l'Archiviste de la Province de Québec.

L'archiviste de la Province de Québec a publié, dans un de ses rapports annuels un Inventaire de la correspondance de Mgr J.-Olivier Briand, 1741-1794. Cet inventaire couvre un grand nombre de lettres de $\mathrm{Mgr}$ Briand à ses prêtres, aux vicaires-généraux, au gouverneur, etc.... Il nous permet de retracer assez facilement d'autres lettres adressées à Mgr Briand par ces personnes, et dont l'inventaire n'a pas été fait. Le rapport dans lequel on trouve cet inventaire est celui de 1929-30, pp. 47-136 (47-113).

Un autre rapport du même archiviste, celui de 194748 , nous donne aussi un Inventaire de la correspondance de cinq vicaires-généraux avec les évêques de Québec, 1761-1816. On y trouve aussi une vingtaine de lettres de Mgr Briand à M. Marchand, curé de Boucherville et vicaire-général, pp. 71-133 (79-111).

IV - Etudes

a) études de droit ecclésiastique et paroissial. 
Hector Langevin, Droit administratif ou manuel des Paroisses et Fabriques. Québec, Desbarats et Derbishire, $1863.205 \mathrm{p} .22 \mathrm{~cm}$.

Mgr Desautels, Manuel des curés pour le bon gouvernement temporel des fabriques dans le Bas-Canada, etc...., etc...., avec un chapitre sur la dîme. Montréal, John Lovell, 1864. XIII-288p. $17 \mathrm{~cm}$.

J.-U. Baudry, Code des Curés, Marguilliers et Paroissiens accompagné de notes historiques et critiques. Publié sous les auspices de la Société Historique de Montréal. Montréal, des presses à vapeur de la Minerve, 1870 . $303 \mathrm{p} .22 \mathrm{~cm}$.

S. Pagnuelo, Etudes historiques et légales sur la Liberté Religieuse en Canada. Montréal, Beauchemin et Valois, 1872. (18) $-\mathrm{X}-409 \mathrm{p} .22 .5 \mathrm{~cm}$.

Edmond Lareau, Histoire du Droit canadien depuis les origines jusqu'à nos jours. Montréal, Librairie générale de droit et de jurisprudence, A. Périard, libraire-éditeur, 1888 . 2 vol. X-518-544p. $21.5 \mathrm{~cm}$.

P.-B. Mignault, Le Droit paroissial étant une étude historique et légale de la paroisse catholique, de sa création, de son gouvernement et de ses biens et un commentaire raisonné du titre IX des Statuts refondus de la Province de Québec «Du Culte religieux » avec un choix de formules et une table analytique des matières. Montréal, Beauchemin \& Fils, 1893. XXVII-690p. $22 \mathrm{~cm}$.

Emile Chartier, Notre droit ecclésiastique sous le régime français, 5ème partie: La Question de la Dîme. dans BRH, 30 (1924), p. 360-363.

J.-François Pouliot, Traité de Droit fabricien et paroissial. Etude critique et législation comparée. Montréal, Wilson \& Lafleur Limitée, 1936. XVII-1010p. $22.5 \mathrm{~cm}$.

Mgr Paul Bernier, Cours de Droit paroissial et de droit ecclésiastique québécois. Plan général du cours et résumé de chaque chapitre. (ad usum privatum auditorum). Québec-Ottawa, 1946, en lithographie, 34p.

b) études spéciales sur la dîme.

E. de Bellefeuille, Deux questions de dîmes, dans la Revue Canadienne, vol. III(1866), p. 602-617. Il 
s'agit de l'inamovibilité des cures par rapport à la dîme et de la portion congrue de 500 livres établie sous le régime français.

Abbé Auguste Gosselin, Un épisode de l'histoire de la dîme au Canada, 1705-170\%, dans MSRC, $2^{\text {eme }}$ série, vol. IX (1903), section 1, pp. 45-63.

Mgr L.-A. Paquet, La Dîme, dans MSRC, $3^{\text {ème }}$ série, vol. V (1911), section 1, pp. 3-17. 